\title{
MicroRNA-302 inhibits cell migration and invasion in cervical cancer by targeting DCUN1D1
}

\author{
YONGJIE JIANG ${ }^{1}$, RUIJIE HOU ${ }^{1}$, SHAOPING LI ${ }^{1}$, SHAORU LI ${ }^{1}$ and GE DANG ${ }^{2}$ \\ ${ }^{1}$ Department of Gynecology and Obstetrics and ${ }^{2}$ Operating Theatre, \\ The First Affiliated Hospital of Xinxiang Medical University, Weihui, Henan 453100, P.R. China
}

Received July 20, 2017; Accepted October 5, 2017

DOI: $10.3892 /$ etm.2018.6223

\begin{abstract}
MicroRNA serve crucial roles in a variety of human cancer types. The miR-302-367 cluster has been reported to suppress the proliferation of cervical carcinoma cells through the novel target AKT1; however, the molecular mechanism of miR-302 in cervical cancer metastasis remains unclear. The present study aimed to investigate the clinical significance of miR-302-3p expression in cervical cancer, and to examine the regulatory mechanism of miR-302-3p in the malignant phenotypes of cervical cancer cells. The present data indicated that miR-302-3p was significantly downregulated in cervical cancer tissues compared with the level in adjacent non-tumor tissues, and low expression of miR-302-3p was significantly associated with node metastasis, advanced clinical stage, and poor prognosis in patients with cervical cancer. Restoration of miR-302-3p expression caused a significant reduction in cervical cancer cell migration and invasion. Defective in cullin neddylation 1 domain containing 1 (DCUN1D1) was identified as a novel target gene of miR-302-3p, and miR-302-3pnegatively regulated the mRNA and protein expression of DCUN1D1 in cervical cancer HeLa cells. Additionally, overexpression of DCUN1D1 rescued the effects of miR-302-3p on the migration and invasion of cervical cancer cells. Furthermore, DCUN1D1 was upregulated in cervical cancer tissues compared with the levels in adjacent tissues, and its high expression was associated with node metastasis, advanced clinical stage, and shorter survival time in patients with cervical cancer. Notably, a negative correlation between miR-302-3p and DCUN1D1 expression in cervical cancer tissues was observed. Taken together, the present study suggests that miR-302-3p serves a suppressive role in cervical cancer metastasis, partly at least, via directly targeting DCUN1D1. Therefore, miR-302-3p/DCUN1D1 may be a potential therapeutic target for cervical cancer treatment.
\end{abstract}

Correspondence to: Dr Yongjie Jiang, Department of Gynecology and Obstetrics, The First Affiliated Hospital of Xinxiang Medical University, 88 Jiankang Road, Weihui, Henan 453100, P.R. China E-mail: xxjiangyongjie@163.com

Key words: cervical cancer, microRNA, defective in cullin neddylation 1 domain containing 1 , migration, invasion

\section{Introduction}

Cervical cancer is one of the most common cancer types in women worldwide, and the majority of cases occur in developing countries, such as China (1). The poor prognosis of patients with advanced cervical cancer is largely attributed to intrinsic molecular changes $(2,3)$. During the growth and metastasis of cervical cancer, various oncogenes and tumor suppressors have been demonstrated to serve key roles, and some of these are suggested to become potential therapeutic targets for cancer treatment (4-6).

MicroRNA (miRNA) are a class of small non-coding RNA $(7,8)$. They directly bind to the 3 ' untranslated region (UTR) of their target mRNA, and cause translation repression or mRNA degradation; thus, they are important regulators of gene expression $(9,10)$. Various miRNA have been demonstrated to be involved in a variety of cellular biological processes, including cell proliferation, differentiation, survival, migration and invasion (8,11-13). Additionally, a large number of miRNA, including miR-181 (14), -186 (15), -126 (16) and -424 (17), have been reported to serve promoting or suppressive roles in tumor growth and metastasis of various human malignances, such as cervical cancer.

Among these miRNA, miR-302-3p is a member of the miR-302-3p/367 cluster and acts as a tumor suppressor in several cancer types $(18,19)$. For instance, miR-302-3p may inhibit the tumorigenicity of endometrial cancer cells by suppressing cyclin D1 and cyclin-dependent kinase 1 (19). In addition, forced expression of miR-302-3p suppresses tumorigenic gene expression patterns in glioblastoma cells and abolishes transformation-related phenotypes (18). A study by Cai et al (20) reported that the miR-302-367 cluster suppresses the proliferation of cervical carcinoma cells through the novel target AKT1. However, the regulatory mechanism of miR-302-3p underlying cervical cancer metastasis remains largely unknown.

Defective in cullin neddylation 1 domain containing 1 (DCUN1D1) serves as an accessory E3 in neddylation by binding to cullin and Ubc12 to allow efficient transfer of Nedd8, and promotes nuclear translocation and assembly of the neddylation E3 complex (21). Recently, miR-218 was reported to inhibit cervical cancer cell migration and invasion by targeting DCUN1D1 (22). However, whether DCUN1D1 is also regulated by other miRNA in cervical cancer remains unclear. 
Therefore, the present study aimed to investigate the clinical significance of miR-302-3p expression in cervical cancer. The regulatory mechanism of miR-302-3p in the malignant phenotypes of cervical cancer cells was also examined.

\section{Materials and methods}

Clinical tissue samples. Cervical cancer tissues and matched adjacent normal tissues were collected from 68 patients with cervical cancer at the First Affiliated Hospital of Xinxiang Medical University (Weihui, China) between September 2010 and May 2012. The age range of the patients was 35-68 years, with a mean age of 61.6 years. The clinicopathological characteristics of the patients are summarized in Table I. The patients did not receive radiation therapy or chemotherapy prior to surgery. The present study was approved by the Ethics Committee of the First Affiliated Hospital of Xinxiang Medical University, and written informed consent was obtained from all patients. All tissues were placed in liquid nitrogen immediately after surgical resection and stored at $-80^{\circ} \mathrm{C}$ before use.

Cell culture. The human cervical cancer HeLa cell line was purchased from the Cell Bank of Chinese Academy of Sciences (Shanghai, China). Cells were cultured in Dulbecco's modified Eagle's medium (DMEM; Thermo Fisher Scientific, Inc., Waltham, MA, USA) supplemented with $10 \%$ fetal bovine serum (FBS; Thermo Fisher Scientific, Inc.) at $37^{\circ} \mathrm{C}$ in a humidified atmosphere containing $5 \% \mathrm{CO}_{2}$.

Cell transfection. For miR-302-3p and DCUN1D1 function analysis, HeLa cells were transfected with $100 \mu \mathrm{M}$ negative control (NC) miR (cat. no. CmiR0001; Guangzhou Fulengen Co., Ltd., Guangzhou, China), $100 \mu \mathrm{M}$ miR-302-3p mimic (5'-uaagugcuuccauguuuugguga-3'; Guangzhou Fulengen Co., Ltd.), or co-transfected with $100 \mu \mathrm{M}$ miR-302-3p mimic and $100 \mu \mathrm{M}$ of pcDNA3.1-DCUN1D1 open reading frame (ORF) plasmid (Yearthbio, Changsha, China), or co-transfected with $100 \mu \mathrm{M}$ miR-302-3p mimic and 100 ng blank pcDNA3.1 vector. Transfection was performed using Lipofectamine 2000 (Thermo Fisher Scientific, Inc.), according to the manufacturer's protocol. Following transfection for $48 \mathrm{~h}$, the expression of miR-302-3p or DCUN1D1 was evaluated.

Reverse transcription-quantitative polymerase chain reaction (RT-qPCR). Total RNA was extracted from tissues and cells using TRIzol reagent (Thermo Fisher Scientific, Inc.). For miRNA expression detection, an Mir-X ${ }^{\mathrm{TM}}$ miRNA qRT-PCR SYBR $^{\circledR}$ kit (Clontech Laboratories, Inc., Mountainview, CA, USA) was utilized to perform RT-qPCR, according to the manufacturer's protocol. U6 was used as an internal reference. For mRNA expression detection, a OneStep RT-PCR kit (Qiagen, Inc., Valencia, CA, USA) was used to perform RT-qPCR, according to the manufacturer's recommendations. GAPDH was used as an internal reference. The primer sequences used were as follows: miR-302-3p forward, 5'-TAA GTGCTTCCATGTTTTGGTGA-3' and reverse, 5'-GAACAT GTCTGCGTATCTCAGACTTC-3'; U6 forward, 5'-GCTTCG GCAGCACATATA-3' and reverse, 5'-AACGCTTCACGA ATTTGCGT-3'; DCUN1D1 forward, 5'-TGCCTACTGGAA CTTAGTGCT-3' and reverse, 5'-CTGCAATCATCGTAC
TGAAGTCT-3'; and GAPDH forward, 5'-ACAACTTTGGTA TCGTGGAAGG-3' and reverse, 5'-GCCATCACGCCACAG TTTC-3'. The reaction conditions were as follows: $95^{\circ} \mathrm{C}$ for $3 \mathrm{~min}$, followed by 40 cycles of $95^{\circ} \mathrm{C}$ for $30 \mathrm{sec}$ and $60^{\circ} \mathrm{C}$ for $30 \mathrm{sec}$. The relative expression was analyzed using the $2^{-\Delta \Delta \mathrm{Cq}}$ method (23).

Cell migration analysis. HeLa cells were cultured to full confluence in a 6 -well plate $\left(5 \times 10^{5}\right.$ cells/well). The cell monolayer was scraped with a $200-\mu 1$ pipette tip, generating a wound $\sim 1 \mathrm{~mm}$ wide. Following three washes with DMEM, cells were incubated in DMEM supplemented $10 \%$ FBS at $37^{\circ} \mathrm{C}$. After $48 \mathrm{~h}$, cells were photographed under a light microscope (Olympus Corporation, Tokyo, Japan) at magnification x40.

Cell invasion analysis. A Matrigel pre-coated Transwell chamber (BD Biosciences, Franklin Lakes, NJ, USA) was used to study cell invasion. A HeLa cell suspension $\left(3 \times 10^{5}\right.$ cells $\left./ \mathrm{ml}\right)$ was prepared in DMEM, which was added into the upper chamber. Following this, $300 \mu \mathrm{l}$ DMEM supplemented with $10 \%$ FBS was added into the lower chamber. After incubation at $37^{\circ} \mathrm{C}$ for $24 \mathrm{~h}$, the cells not invading through the membrane in the filter were wiped out carefully with a cotton-tipped swab, while the cells invading through the membrane were stained with $0.1 \%$ crystal violet (Beyotime Institute of Biotechnology, Haimen, China) for $10 \mathrm{~min}$ at room temperature. The invading cells were counted under a light microscope (Olympus Corporation) at magnification $\mathrm{x} 400$.

Bioinformatics prediction and dual-luciferase reporter gene assay. TargetScan online software was used to predict the potential target genes of miR-302-3p (24). The wild type (WT) of DCUN1D1 3'UTR containing the binding sites of miR-302-3p or the mutant (MT) type of DCUN1D1 3'UTR lacking the binding sites of miR-302-3p was amplified, which was then subcloned into the downstream of the Renilla luciferase gene in the psiCHECK-2 vector (Promega Corporation, Madison, WI, USA). HeLa cells were co-transfected with WT or MT DCUN1D1 3'UTR luciferase reporter gene plasmid, and miR-302-3p mimic or NC, using Lipofectamine 2000 according to the manufacturer's protocol. After $48 \mathrm{~h}$, the cells were assayed for luciferase activity using a Dual-Luciferase Reporter Assay System (Promega Corporation). The firefly luciferase activities were normalized to Renilla luciferase activity.

Western blot analysis. Tissues and cells were lysed in cold radioimmunoprecipitation assay buffer (Beyotime Institute of Biotechnology), and the protein concentration was determined using a BCA Protein Assay kit (Pierce; Thermo Fisher Scientific, Inc.), according to the manufacturer's protocol. A total of $80 \mu \mathrm{g}$ protein was separated using $12 \%$ SDS-PAGE and transferred to a polyvinylidene fluoride (PVDF) membrane (Thermo Fisher Scientific, Inc.). The membrane was subsequently blocked in 5\% non-fat dried milk in PBS (Thermo Fisher Scientific, Inc.) at room temperature for $3 \mathrm{~h}$. Following three washes with PBS, the membrane was incubated with rabbit anti-human DCUN1D1 antibody (1:50; ab181233; Abcam, Cambridge, MA, USA) or rabbit anti-human GAPDH antibody (1:50; ab9485; Abcam) for $3 \mathrm{~h}$ at room temperature. 
Table I. Association between miR-302-3p expression and clinicopathological characteristics of patients with cervical cancer.

miR-302-3p expression level

\begin{tabular}{|c|c|c|c|c|}
\hline \multirow[b]{2}{*}{ Variables } & \multirow[b]{2}{*}{ Cases $(n=68)$} & & \multirow[b]{2}{*}{ P-value } \\
\hline & & Low $(n=39)$ & High $(n=29)$ & \\
\hline Age, years & & & & 0.799 \\
\hline$<55$ & 24 & 13 & 11 & \\
\hline$\geq 55$ & 44 & 26 & 18 & \\
\hline Tumor size, cm & & & & 0.466 \\
\hline$\leq 4$ & 41 & 22 & 19 & \\
\hline$>4$ & 27 & 17 & 10 & \\
\hline Differentiation & & & & 0.150 \\
\hline Well-moderate & 52 & 27 & 25 & \\
\hline Poor & 16 & 12 & 4 & \\
\hline Clinical stage & & & & 0.041 \\
\hline I-II & 44 & 21 & 23 & \\
\hline III-IV & 24 & 18 & 6 & \\
\hline Lymph node metastasis & & & & 0.019 \\
\hline No & 45 & & 24 & \\
\hline Yes & 23 & & 5 & \\
\hline Distant metastasis & & & & 0.068 \\
\hline No & 59 & 31 & 28 & \\
\hline Yes & 9 & & 1 & \\
\hline
\end{tabular}

Following three washes with PBS, the membrane was incubated with horseradish peroxidase conjugated goat anti-rabbit secondary antibody (1:5,000; ab6721, Abcam) for $40 \mathrm{~min}$ at room temperature. Following three washes with PBS, the immune complex on the PVDF membrane was detected using an Enhanced Chemiluminescence Western Blotting kit (Pierce; Thermo Fisher Scientific, Inc.). The protein expression was determined using Image-Pro Plus software 6.0 (Media Cybernetics, Inc., Rockville, MD, USA), and GAPDH was used as an internal control.

Statistical analysis. Data were presented as the mean \pm standard deviation. SPSS version 17.0 (SPSS, Inc., Chicago, IL, USA) was used to perform statistical analysis. Differences were analyzed using a Student's t-test for two-group comparison, or one-way analysis of variance for comparison of more than two groups with Turkey's post hoc test. The association between the gene expression and clinicopathological characteristics was examined using the $\chi^{2}$ test. The Kaplan-Meier method was used to conduct survival analysis. Pearson correlation analysis was performed to examine the correlation between the miR-302-3p and DCUN1D1 expression. $\mathrm{P}<0.05$ was considered to indicate a statistically significant difference.

\section{Results}

miR-302-3p is downregulated in cervical cancer. In the present study, RT-qPCR data demonstrated that the miR-302-3p expression level was significantly reduced in cervical cancer tissues compared with that in adjacent non-tumor tissues (Fig. 1A). The patients with cervical cancer were subsequently divided into a high miR-302-3p group and low miR-302-3p group based on the mean value of miR-302-3p expression. It was demonstrated that low miR-302-3p expression was significantly associated with advanced clinical stage and node metastasis (Table I), suggesting that downregulation of miR-302-3p may contribute to the malignant progression of cervical cancer. Following this, the association between miR-302-3p expression and the prognosis of patients with cervical cancer was studied. Data of Kaplan-Meier analysis indicated that patients in the low miR-302-3p group had a significantly shorter survival time compared with that of the patients with high miR-302-3p expression (Fig. 1B).

miR-302-3p inhibits cell migration and invasion in cervical cancer. As low expression of miR-302-3p was associated with node metastasis in cervical cancer, the role of miR-302-3p in the regulation of cervical cancer cell migration and invasion was investigated. HeLa cells were transfected with miR-302-3p mimic or scramble miR mimic. RT-qPCR data indicated that the miR-302-3p expression levels were significantly increased in the miR-302-3p group compared with the level in the control group (Fig. 2A). However, transfection with scramble miR mimic did not significantly affect the expression of miR-302-3p in HeLa cells compared with the level in the control group (Fig. 2A). Wound healing and Transwell assays were subsequently conducted to evaluate cell migration and invasion. The present data indicated that overexpression of miR-302-3p significantly inhibited the migration and invasion of HeLa cells compared with that observed in the NC group (Fig. 2B and C). Therefore, miR-302-3p may have suppressive effects on the metastasis of cervical cancer. 
A

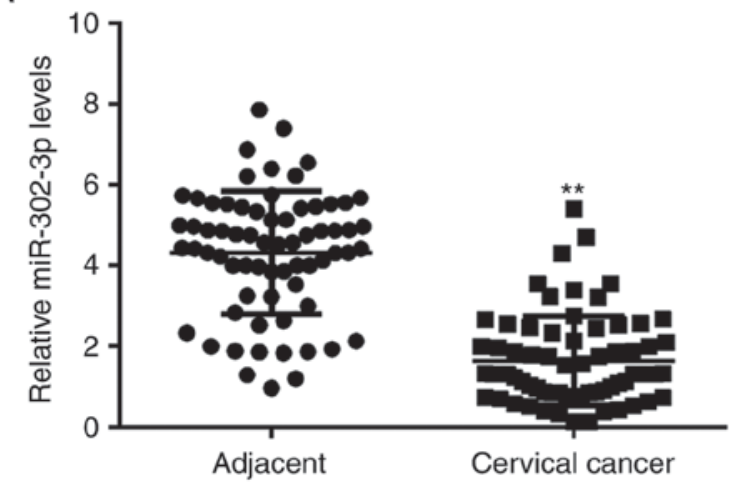

B

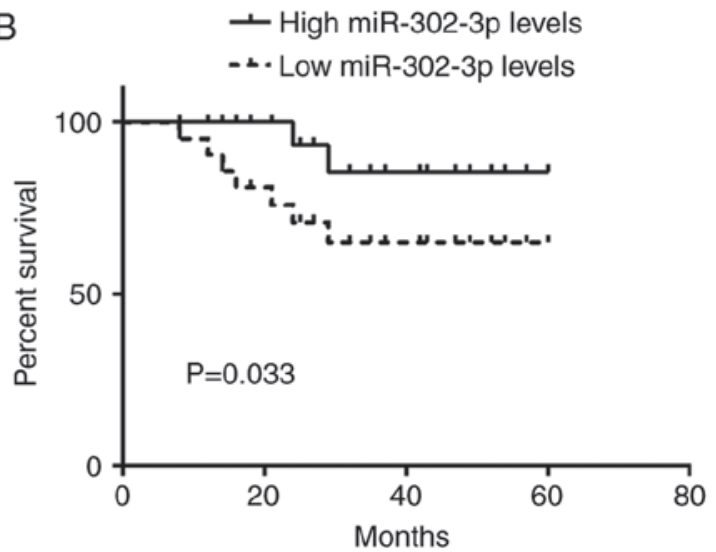

Figure 1. miR-302-3p is downregulated in cervical cancer. (A) Reverse transcription-quantitative polymerase chain reaction was utilized to evaluate the miR-302-3p expression level in cervical cancer tissues and adjacent normal tissues.(B) Low expression of miR-302-3p was associated with a shorter survival time in patients with cervical cancer. ${ }^{* *} \mathrm{P}<0.01$ vs. adjacent tissues.
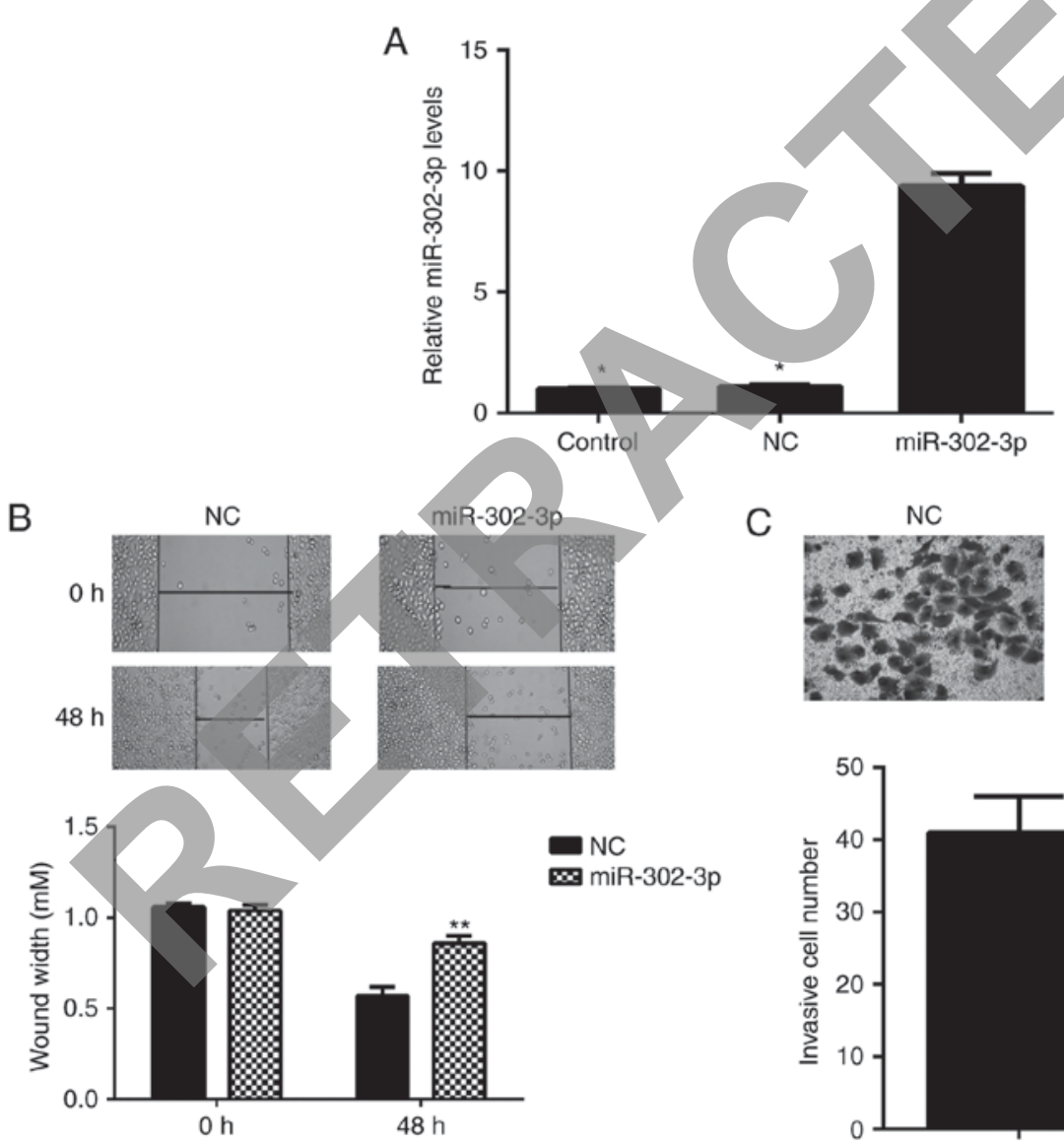

C
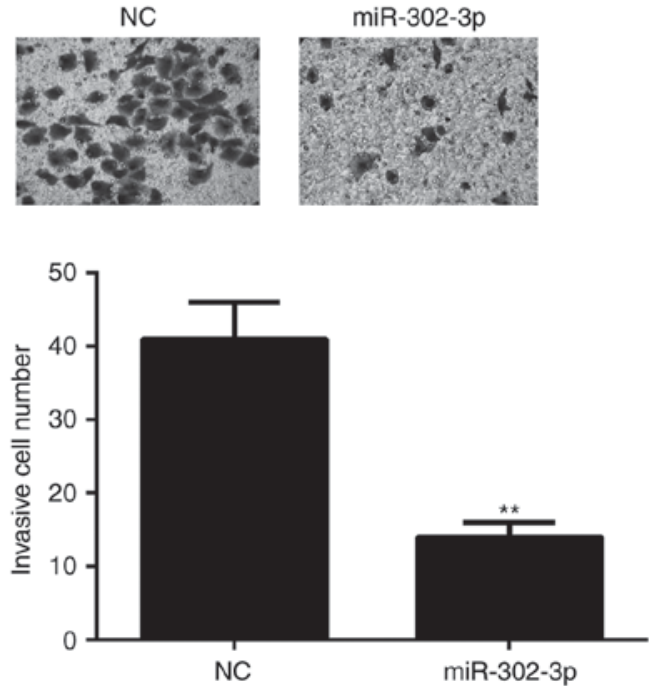

Figure 2. miR-302-3p inhibits cell migration and invasion in cervical cancer. miR-302-3p mimic or NC miR mimic was used to transfect HeLa cells, respectively. (A) Following transfection, reverse transcription-quantitative polymerase chain reaction was used to evaluate the miR-302-3p expression level. (B) Wound healing and (C) Transwell assays were used to examine cell migration and invasion, respectively. ${ }^{*} \mathrm{P}<0.01$ vs. miR-302-3p group; ${ }^{* *} \mathrm{P}<0.01 \mathrm{vs.} \mathrm{NC}$. NC, negative control.

miR-302-3p directly targets DCUNID1 in cervical cancer cells. The potential target genes of miR-302-3p were explored, and TargetScan online software indicated that DCUN1D1 was a putative target gene of miR-302-3p (Fig. 3A). In addition, their target relationship was evolutionally conserved (data not shown). Luciferase reporter plasmids containing the WT and MT type of DCUN1D1 3'UTR were generated
(Fig. 3B). Luciferase reporter gene assay results indicated that the luciferase activity was significantly decreased in the presence of miR-302-3p in HeLa cells transfected with WT DCUN1D1 3'UTR luciferase reporter plasmid compared with the level in the control group. However, there was no significant effect on luciferase activity when transfection with the MT type of DCUN1D1 3'UTR luciferase reporter plasmid was 


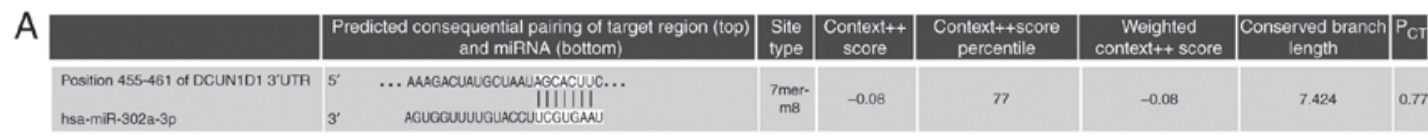

B WT DCUN1D13'UTR 5'... AGACUAUGCUAAUAGCACUUC...3'

miR-302-3p $\quad 3^{\prime}$...GGGAGUUUUUCCUUCGUGAAA...5'

MT DCUN1D1 3'UTR $\quad 5^{\prime}$...AGACUAUGCUAAUAGGUGAUC...3'

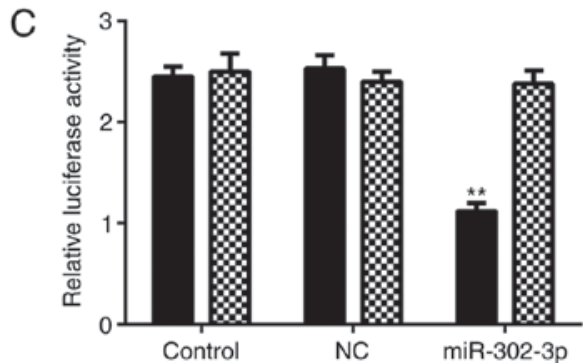

WT DCUN1D1 3'UTR
$\mathbb{M}$ MT DCUN1D1 3'UTR

Figure 3. miR-302-3p directly targets DCUN1D1 in cervical cancer cells. (A) DCUN1D1 is a putative target gene of miR-302-3p. (B) The WT or MT types of DCUN1D1 3'UTR luciferase reporter plasmids were generated. (C) The luciferase activity was significantly decreased in the presence of miR-302-3p in HeLa cells transfected with WT DCUN1D1 3'UTR luciferase reporter plasmid, but not reduced when transfected with the MT type DCUN1D1 3'UTR luciferase reporter plasmid. "* $\mathrm{P}<0.01$ vs. WT control. WT, wild type; MT, mutant; NC, negative control; UTR, untranslated region; DCUN1D1, defective in cullin neddylation 1 domain containing 1 .

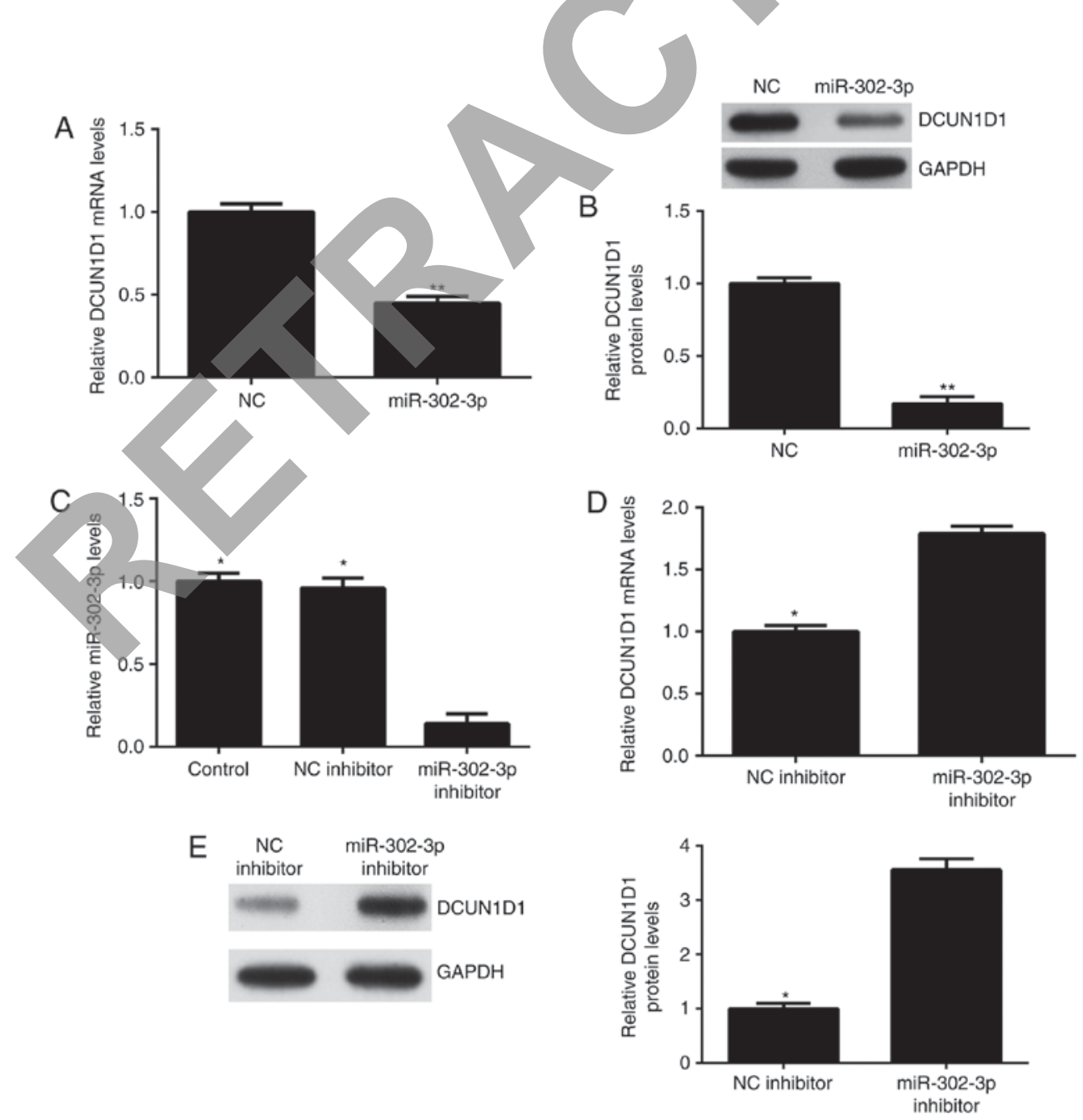

Figure 4. DCUN1D1 expression is negatively regulated by miR-302-3p in HeLa cells. The (A) mRNA and (B) protein expression levels of DCUN1D1 were examined in HeLa cells transfected with miR-302-3p mimic or scramble miR mimic (NC), respectively. Then, HeLa cells were transfected with NC inhibitor or miR-302-3p inhibitor. (C) After transfection, quantitative real-time PCR was used to evaluate the miR-302-3p expression level. The (D) mRNA and (E) protein expression levels of DCUN1D1 were also evaluated. ${ }^{* *} \mathrm{P}<0.01$ vs. NC; ${ }^{*} \mathrm{P}<0.01$ vs. miR-302-3p inhibitor. DCUN1D1, defective in cullin neddylation 1 domain containing $1 ; \mathrm{NC}$, negative control. 
A

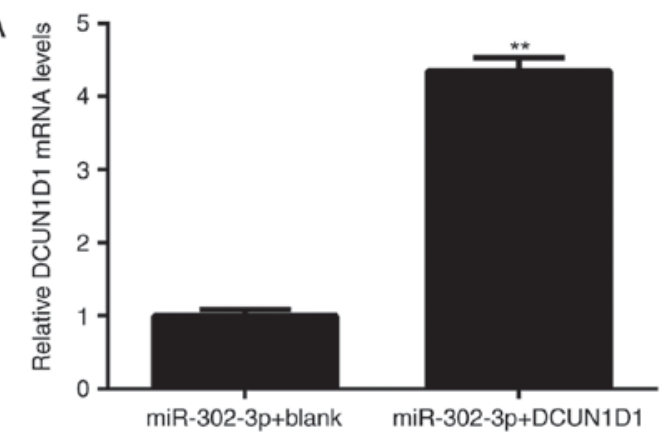

C
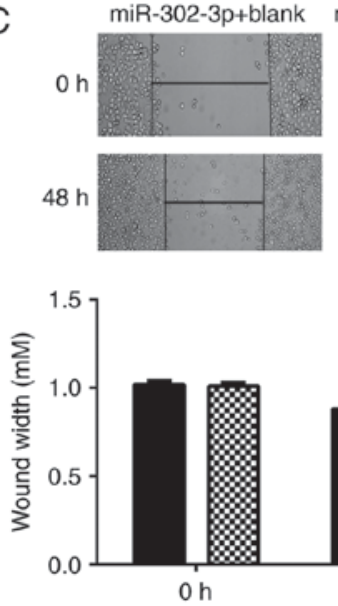

miR-302-3p+DCUN1D1
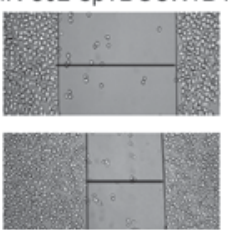

miR-302-3p+blank $\mathbf{m i R}-302-3 p+D C U N 1 D$

B
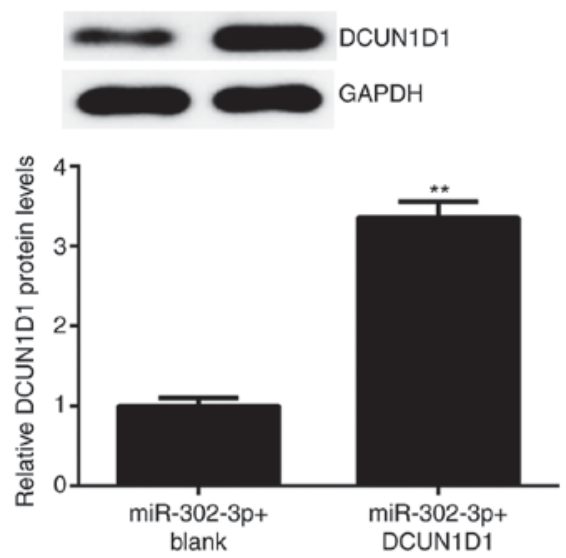

D miR-302-3p+blank miR-302-3p+DCUN1D1
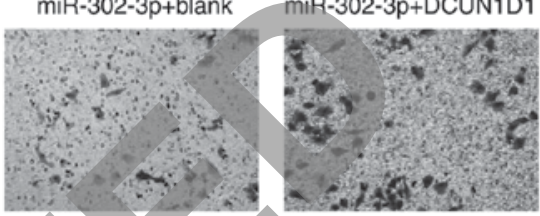

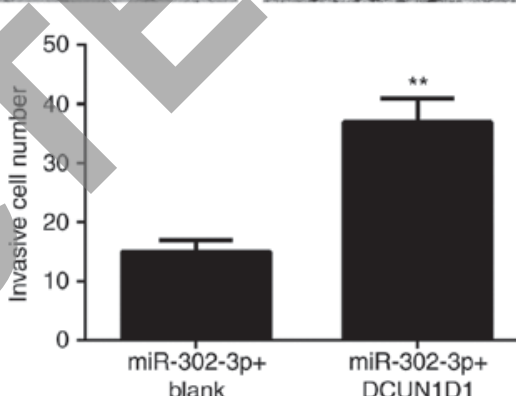

Figure 5. DCUN1D1 reverses the suppressive effects of miR-302-3p on cervical cancer cell migration and invasion. The miR-302-3p-overexpressing HeLa cells were transfected with pcDNA3.1-DCUN1D1 open reading frame plasmid or with blank vector. The (A) mRNA and (B) protein expression levels of DCUN1D1 were examined. (C) Wound healing and (D) Transwell assays were used to examine cell migration and invasion. ${ }^{* *} \mathrm{P}<0.01 \mathrm{vs}$. miR-302-3p + blank. DCUN1D1, defective in cullin neddylation 1 domain containing 1.

conducted (Fig. 3C). These findings indicate that DCUN1D1 is a target gene of miR-302-3p in HeLa cells.

DCUNID1 expression is negatively regulated by $\mathrm{miR}-302-3 p$ in HeLa cells. As miRNA generally negatively regulate the expression of their target genes, the effects of miR-302-3p on DCUN1D1 expression in HeLa cells were explored. As demonstrated in Fig. 4A and B, the mRNA and protein expression levels of DCUN1D1 in HeLa cells were significantly reduced after overexpression of miR-302-3p compared with the levels in the NC group. HeLa cells were transfected with miR-302-3p inhibitor or NC inhibitor, respectively. Following transfection, the miR-302-3p expression level was significantly reduced in the miR-302-3p inhibitor group compared with the level in the control group (Fig. 4C). Transfection with NC inhibitor demonstrated no significant effect on the miR-302-3p expression levels compared with that in the control group (Fig. 4C). mRNA and protein expression levels of DCUN1D1 were revealed to be significantly increased following inhibition of miR-302-3p compared with the levels in the NC inhibitor group (Fig. 4D and E). These data indicate that miR-302-3p could negatively regulate the expression of DCUN1D1 in HeLa cells.

DCUN1D1 reverses the suppressive effects of miR-302-3p on cervical cancer cell migration and invasion. Based on the above findings, it was speculated that DCUN1D1 may be involved in the miR-302-3p-mediated migration and invasion of HeLa cells. To clarify this speculation, the miR-302-3p-overexpressing HeLa cells were transfected with pcDNA3.1-DCUN1D1 ORF plasmid or blank vector. As demonstrated in Fig. 5A and $\mathrm{B}$, following transfection, the mRNA and protein expression levels of DCUN1D1 were significantly higher in the miR-302-3p + DCUN1D1 group compared with the levels in the miR-302-3p + blank group. Furthermore, following transfection, cell migration and invasion were significantly increased in the miR-302-3p + DCUN1D1 group compared with the levels in the miR-302-3p + blank group (Fig. 5C and D). Thus, DCUN1D1 rescued the miR-302-3p-mediated inhibition of HeLa cell migration and invasion.

DCUN1D1 is upregulated in cervical cancer. Finally, the DCUN1D1 expression level in cervical cancer tissues was determined. RT-qPCR and western blotting data indicated that the mRNA and protein expression levels of DCUN1D1 were significantly and markedly increased, respectively, in cervical cancer tissues compared with the levels in adjacent non-tumor tissues (Fig. 6A and B, respectively). In addition, Pearson correlation analysis data demonstrated an inverse correlation between the miR-302-3p and DCUN1D1 expression levels in cervical cancer tissues (Fig. 6C), suggesting that the increased 

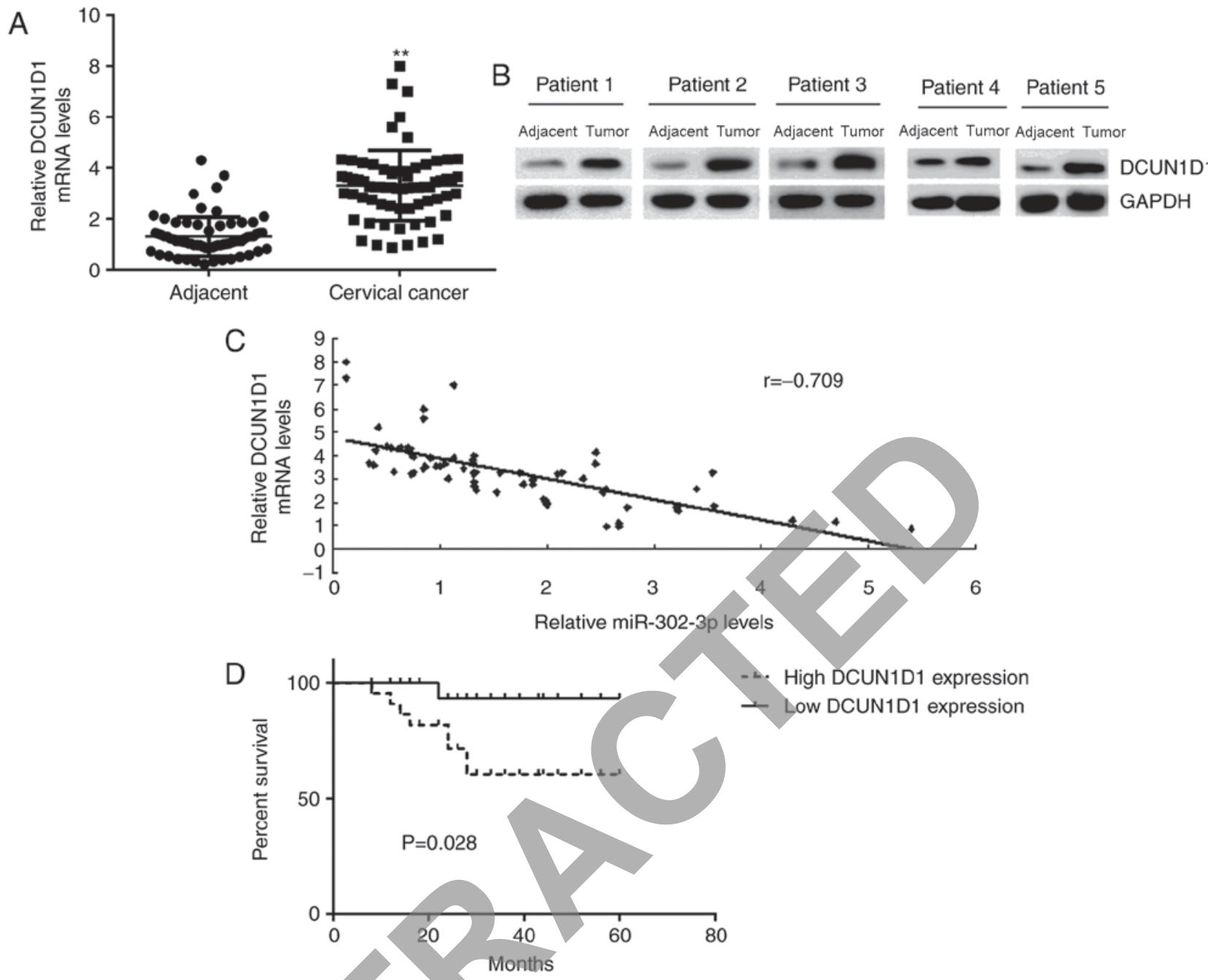

Figure 6. DCUN1D1 is upregulated in cervical cancer. The (A) mRNA and (B) protein expression levels of DCUN1D1 were higher in cervical cancer tissues than in adjacent non-tumor tissues. (C) An inverse correlation existed between miR-302-3p and DCUN1D1 expression in cervical cancer tissues. (D) Patients with cervical cancer and high DCUN1D1 expression demonstrated a shorter survival time than those with those with low expression of DCUN1D1. ${ }^{* *} \mathrm{P}<0.01$ vs. adjacent tissues. DCUN1D1, defective in cullin neddylation 1 domain containing 1.

DCUN1D1 expression in cervical cancer may be due to the downregulation of miR-302-3p.

The patients with cervical cancer were divided into a high DCUN1D1 group and low DCUN1D1 group. As indicated in Table II, high expression of DCUN1D1 was significantly associated with advanced clinical stage and node metastasis in cervical cancer. In addition, the patients with high expression of DCUN1D1 demonstrated a shorter survival time compared with that observed in patients with low DCUN1D1 expression (Fig. 6D). Therefore, upregulation of DCUN1D1 may contribute to the malignant progression and poor prognosis of patients with cervical cancer.

\section{Discussion}

The molecular mechanism of miR-302 in cervical cancer metastasis has not previously been studied. The present study demonstrated that miR-302-3p was significantly downregulated in cervical cancer tissues compared with the levels in adjacent non-tumor tissues, and low expression of miR-302-3p was significantly associated with node metastasis, advanced clinical stage, and poor prognosis in patients with cervical cancer. Restoration of miR-302-3p expression caused a significant reduction in cervical cancer cell migration and invasion. DCUN1D1 was identified as a novel target gene of miR-302-3p, and miR-302-3p negatively regulated the mRNA and protein expression levels of DCUN1D1 in HeLa cells. In addition, overexpression of DCUN1D1 rescued the effects of miR-302-3p on the migration and invasion of cervical cancer cells. Furthermore, DCUN1D1 was upregulated in cervical cancer tissues compared with the levels in adjacent tissues, and its high expression was associated with node metastasis, advanced clinical stage, and shorter survival time of patients with cervical cancer. Notably, a negative correlation between miR-302-3p and DCUN1D1 expression was observed in cervical cancer tissues.

miR-302-3p is a member of the miR-302-3p-367 cluster, which has been demonstrated to be specifically expressed in human embryonic stem cells (25). Additionally, the miR-302-3p-367 cluster has potential to convert somatic cells into induced pluripotent stem cells (26). A study by Cai et al (20) investigated the role of the miR-302-3p-367 cluster in cervical carcinoma. The study indicated that ectopic expression of the miR-302-3p-367 cluster inhibited cervical cancer 
Table II. Association between DCUN1D1 expression and clinicopathological characteristics of patients with cervical cancer.

\begin{tabular}{|c|c|c|c|c|}
\hline \multirow[b]{2}{*}{ Variables } & \multirow[b]{2}{*}{ Cases $(n=68)$} & \multicolumn{2}{|c|}{ DCUN1D1 expression level } & \multirow[b]{2}{*}{ P-value } \\
\hline & & Low expression $(n=31)$ & High expression $(n=37)$ & \\
\hline Age, years & & & & 0.619 \\
\hline$<55$ & 24 & 12 & 12 & \\
\hline$\geq 55$ & 44 & 19 & 25 & \\
\hline Tumor size, $\mathrm{cm}$ & & & & 0.322 \\
\hline$\leq 4$ & 41 & 21 & 20 & \\
\hline$>4$ & 27 & 10 & 17 & \\
\hline Differentiation & & & & 0.085 \\
\hline Well-moderate & 52 & 27 & 25 & \\
\hline Poor & 16 & 4 & 12 & \\
\hline Clinical stage & & & & 0.021 \\
\hline I-II & 44 & 25 & 19 & \\
\hline III-IV & 24 & 6 & 18 & \\
\hline Lymph node metastasis & & & & 0.038 \\
\hline No & 45 & 25 & 20 & \\
\hline Yes & 23 & 6 & 17 & \\
\hline Distant metastasis & & & & 0.033 \\
\hline No & 59 & & 29 & \\
\hline Yes & 9 & 1 & 8 & \\
\hline
\end{tabular}

cell proliferation and tumor formation by inducing a cell cycle arrest at the $\mathrm{G}_{1}$ stage (20). It was further demonstrated that the miR-302-3p-367 cluster suppressed the expression of cyclin D1 and AKT1, while promoted the expression of p27 (Kip1) and p21 (Cip1), which contributed to the inhibition of cervical cancer cell proliferation (20). These findings suggest that miR-302-3p serves a suppressive role in the growth of cervical cancer. However, the clinical significance of miR-302-3p expression, as well as the effects of miR-302-3p on cervical cancer metastasis, have not previously been studied. The present study revealed that miR-302-3p was significantly downregulated in cervical cancer tissues compared with the levels in adjacent non-tumor tissues. In addition, low expression of miR-302-3p was associated with node metastasis and advanced clinical stage. These findings suggest that miR-302-3p may also serve inhibitory roles in the metastasis of cervical cancer. Therefore, cervical cancer cells were transfected with miR-302-3p mimic and it was demonstrated that overexpression of miR-302-3p significantly downregulated the migration and invasion of cervical cancer cells.

As miRNA function through regulating gene expression, potential targets of miR-302-3p in cervical cancer cells were explored by performing bioinformatics analysis. The data of TargetScan online software indicated that DCUN1D1 was a putative target gene of miR-302-3p, which was further confirmed by luciferase reporter gene analysis. Previous studies have demonstrated that DCUN1D1 is upregulated in squamous cell carcinoma, and promotes non-small cell lung cancer progression and brain metastasis $(27,28)$. Recently, Jiang et al (22) reported that miR-218 inhibited epithelial-mesenchymal transition, migration and invasion by targeting Scm-like with four mbt domains 1 and DCUN1D1 in cervical cancer. However, the regulatory mechanism of DCUN1D1 expression in cervical cancer has remained obscure. The present study demonstrated that DCUN1D1 rescued the miR-302-3p-mediated inhibition of cervical cancer cell migration and invasion. Thus, the suppressive effects of miR-302-3p on cervical cancer cells may be through direct targeting of DCUN1D1. Additionally, as the present study demonstrated that DCUN1D1 was significantly upregulated in cervical cancer, and the DCUN1D1 levels were inversely correlated with the miR-302-3p levels, it may be suggested that the upregulation of DCUN1D1 may be attributed to the reduced expression of miR-302-3p in cervical cancer.

To the best of our knowledge, the present study demonstrated, for the first time, that miR-302-3p is significantly downregulated in cervical cancer, and serves a suppressive role in the migration and invasion of cervical cancer cells, partly at least, through directly targeting DCUN1D1. These findings suggest that the miR-302-3p/DCUN1D1 axis may be a potential therapeutic target for the treatment of cervical cancer.

\section{Competing interests}

The authors declare that they have no competing interests.

\section{References}

1. Torre LA, Bray F, Siegel RL, Ferlay J, Lortet-Tieulent J and Jemal A: Global cancer statistics, 2012. CA Cancer J Clin 65: 87-108, 2015 
2. Li Y, Li S and Huang L: Knockdown of Rap2B, a Ras superfamily protein, inhibits proliferation, migration, and invasion in cervical cancer cells via regulating the ERK1/2 signaling pathway. Oncol Res 26: 123-130, 2018.

3. Luo C and Qiu J: MiR-181a inhibits cervical cancer development via downregulating GRP78. Oncol Res 25: 1341-1348, 2017.

4. Zhang X, Cai D, Meng L and Wang B: MicroRNA-124 inhibits proliferation, invasion, migration and epithelial-mesenchymal transition of cervical carcinoma cells by targeting astrocyteelevated gene-1. Oncol Rep 36: 2321-2328, 2016.

5. Zeng F, Xue M, Xiao T, Li Y, Xiao S, Jiang B and Ren C: MiR-200b promotes the cell proliferation and metastasis of cervical cancer by inhibiting FOXG1. Biomed Pharmacother 79: 294-301, 2016.

6. Yao J, Deng B, Zheng L, Dou L, Guo Y and Guo K: miR-27b is upregulated in cervical carcinogenesis and promotes cell growth and invasion by regulating CDH11 and epithelial-mesenchymal transition. Oncol Rep 35: 1645-1651, 2016.

7. Ambros V: The functions of animal microRNAs. Nature 431: 350-355, 2004

8. Bartel DP: MicroRNAs: Genomics, biogenesis, mechanism, and function. Cell 116: 281-297, 2004.

9. Chen X and Chen J: MiR-3188 regulates cell proliferation, apoptosis, and migration in breast cancer by targeting TUSC5 and regulating the p38 MAPK signaling pathway. Oncol Res: May 26, 2017 (Epub ahead of print).

10. Ji S, Zhang B, Kong Y, Ma F and Hua Y: MiR-326 inhibits gastric cancer cell growth through down regulating NOB1. Oncol Res 25: 853-861, 2017.

11. Jiang Z, Zhang Y, Cao R, Li L, Zhong K, Chen Q and Xiao J: MiR-5195-3p inhibits proliferation and invasion of human bladder cancer cells by directly targeting oncogene KLF5. Oncol Res 25: 1081-1087, 2017.

12. Li H, Xiang Z, Liu Y, Xu B and Tang J: MicroRNA-133b inhibits proliferation, cellular migration, and invasion via targeting LASP1 in hepatocarcinoma cells. Oncol Res 25: 1269-1282, 2017.

13. Li X, Li Y and Lu H: MiR-1193 suppresses proliferation and invasion of human breast cancer cells through directly targeting IGF2BP2. Oncol Res 25: 579-585, 2017.

14. Yang M,Zhai X, Ge T, Yang C and Lou G: MiR-181a-5p promotes proliferation and invasion, and inhibits apoptosis of cervical cancer Cells via regulating inositol polyphosphate-5-phosphatase A (INPP5A). Oncol Res: Jun 23, 2017 (Epub ahead of print).

15. Zhang JJ, Wang DD, Du CX and Wang Y: Long noncoding RNA ANRIL promotes cervical cancer development by acting as a sponge of miR-186. Oncol Res. May 22, 2017 (Epub ahead of print).
16. Wang C, Zhou B, Liu M, Liu Y and Gao R: miR-126-5p Restoration promotes cell apoptosis in cervical cancer by targeting Bcl212. Oncol Res 25: 463-470, 2017.

17. Gao YL, Zhao ZS, Zhang MY, Han LJ, Dong YJ and Xu B: Long noncoding RNA PVT1 facilitates cervical cancer progression via negative regulating of miR-424. Oncol Res 25: 1391-1398, 2017.

18. Yang CM, Chiba T,BrillB, Delis N, von Manstein V, Vafaizadeh V, Oellerich T and Groner B: Expression of the miR-302/367 cluster in glioblastoma cells suppresses tumorigenic gene expression patterns and abolishes transformation related phenotypes. Int $\mathrm{J}$ Cancer 137: 2296-2309, 2015.

19. Yan GJ, Yu F, Wang B, Zhou HJ, Ge QY, Su J, Hu YL, Sun HX and Ding LJ: MicroRNA miR-302 inhibits the tumorigenicity of endometrial cancer cells by suppression of Cyclin D1 and CDK1. Cancer Lett 345: 39-47, 2014.

20. Cai N, Wang YD and Zheng PS: The microRNA-302-367 cluster suppresses the proliferation of cervical carcinoma cells through the novel target AKT1. RNA 19: 85-95, 2013.

21. Huang G, Kaufman AJ, Ramanathan Y and Singh B: SCCRO (DCUN1D1) promotes nuclear translocation and assembly of the neddylation E3 complex. J Biol Chem 286: 10297-10304, 2011.

22. Jiang Z, Song Q, Zeng R, Li J, Li J, Lin X, Chen X, Zhang J and Zheng Y: MicroRNA-218 inhibits EMT, migration and invasion by targeting SFMBT1 and DCUN1D1 in cervical cancer. Oncotarget 7: 45622-45636, 2016.

23. Livak KJ and Schmittgen TD: Analysis of relative gene expression data using real-time quantitative PCR and the 2(-Delta Delta C(T)) method. Methods 25: 402-408, 2001.

24. Agarwal V, Bell GW, Nam JW and Bartel DP: Predicting effective microRNA target sites in mammalian mRNAs. Elife 4, 2015.

. Gao Z, Zhu X and Dou Y: The miR-302/367 cluster: A comprehensive update on its evolution and functions. Open Biol 5: 150138,2015

26. Sandmaier SE and Telugu BP: MicroRNA-mediated reprogramming of somatic cells into induced pluripotent stem cells. Methods Mol Biol 1330: 29-36, 2015.

27. Sarkaria I, O-charoenrat P, Talbot SG, Reddy PG, Ngai I, Maghami E, Patel KN, Lee B, Yonekawa Y, Dudas M, et al: Squamous cell carcinoma related oncogene/DCUN1D1 is highly conserved and activated by amplification in squamous cell carcinomas. Cancer Res 66: 9437-9444, 2006.

28. Yoo J, Lee SH, Lym KI, Park SY, Yang SH, Yoo CY, Jung JH, Kang SJ and Kang CS: Immunohistochemical expression of DCUN1D1 in non-small cell lung carcinoma: Its relation to brain metastasis. Cancer Res Treat 44: 57-62, 2012. 